\title{
IS THERE WATER ICE IN THE LUNAR POLAR CRATERS?
}

\begin{abstract}
Tianxi Sun (孙天锡)*
Accepted 00th January 20xx

This literature review found that it is doubtful that there is water ice in the polar craters on the Moon. In the course of this review, the following findings were found: (1) The absorption strength of hydroxyl radicals and hydroxyl groups are all $2.9 \mu \mathrm{m}$, so it is easy to confuse hydroxyl radicals and hydroxyl groups when interpreting $\mathrm{M}^{3}$ spectra data. I do not doubt the ability of LCROSS to detect $\mathrm{OH}$ from water, but only suspect that LCROSS is unable to distinguish between hydroxyl radicals from water ice and hydroxyl groups from Moon's methanol due to ignore their spectral identity; (2) The water brought by comets and asteroids and the one caused by solar wind has been exhausted by reacts with the widespread methanol on the Moon in the presence of Pt/a-MoC or Pt/C catalysts. These reacts form large amount of hydrogen, thus clarifying a question NASA raised that "Scientists have long speculated about the source of vast quantities of hydrogen that have been observed at the lunar poles"; (3) The vast quantities of hydrogen in lunar polar craters at extremely low temperatures might be in liquid or solid state now, easy to confuse with water ice. It seems that all our previous misconceptions about water ice in the lunar polar craters might be due to the neglect of the widespread chemical role of lunar methanol. It is necessary to conduct in-depth research in this field in the future.
\end{abstract}

\section{Introduction}

A theory that there is water ice in the lunar polar craters is an overwhelming popular theory in the world at present. In the course of unfolding this literature review, I found that it is doubtful that there is water ice in the polar craters on the Moon. The presence of water ice in the lunar polar craters should be worthy of renewed scrutiny.

Now, let's start the debate about whether the Moon has water ice.

Studies of lunar samples and lunar meteorites have found no evidence of the presence of water on the Moon (Ouyang 2005).

Moreover, no water was involved in the formation of the Moon: (1) The main lunar rocks are anorthitite, basalt and breccia, and no aqueous rocks such as sandstone, shale and limestone which are very common on our Earth (Ouyang 2005); Moreover, "as a whole, the $\mathrm{M}^{3}$ data do not provide clear evidence for magmatic water in the anorthositic crust" (Li \& Milliken 2017). (2) Lunar minerals are generally devoid of water. Elements in lunar minerals are all lowvalence, for example, divalent or zero valences for iron. This shows

\footnotetext{
* Suzhou Ecological Environment Bureau, Suzhou 215002, China.

E-mail: 18913505350@163.com

† Footnotes relating to the title and/or authors should appear here.

Electronic Supplementary Information (ESI) available: [details of any supplementary information available should be included here]. See
} DOI: $10.1039 / x 0 \times x 00000 x$ that lunar minerals were formed under a strict reducing environment of no water. The main lunar minerals are pyroxene $\left[(\mathrm{Ca}, \mathrm{Fe}, \mathrm{Mg})_{2} \mathrm{Si}_{2} \mathrm{O}_{6}\right], \quad$ anorthite $\quad\left[(\mathrm{Ca}, \mathrm{Na})(\mathrm{Al}, \mathrm{Si})_{4} \mathrm{O}_{8}\right], \quad$ olivine $\left[(\mathrm{Mg}, \mathrm{Fe})_{2} \mathrm{SiO}_{4}\right]$, ilmenite $\left(\mathrm{FeTiO}_{3}\right)$ and spinel $\left(\mathrm{MgAl}_{2} \mathrm{O}_{4}\right)$; No primary and secondary hydrated minerals (such as clay, mica and amphibole) were found (Ouyang 2005). However, on this point, there is still some debates, for example, recent re-analyses of lunar samples (apatite in basalts) have measured indigenous water (Tartèse et al. 2014; Barnes et al. 2013; Barnes et al. 2014). But, crystalline water normally can not be separated from minerals and is not the water resources we crave. So the lunar crystalline water is meaningless for discussing whether the Moon has water. In addition, people have reason to suspect that the "apatite water" found from their reanalyses is not from the late water pollution?! (3) The absence of water in the lunar rock profile (Matthewman et al. 2015). (4) The Moon is largely anhydrous (Matthewman et al. 2015). (5) Evidence from Apollo and Luna samples indicate that lunar materials are deficient in volatiles compared with Earth (Lucey et al. 2006). (6) There have not existed any traces of flowing water on the Moon. On the contrary, there are a lot of traces of flowing water on the Mars (ESA 2015; ESA 2017).

In addition, the high temperature generated by impacting on the Moon, coupled with the lack of atmosphere and low gravity, make it difficult to preserve water on lunar surface.

So it is necessary to reexamine whether there is water ice in the lunar polar craters.

\section{Methodology}


Contrary to the convention of review for review, this literature review closely revolves around the theme of the paper "Is there water ice in the Lunar polar craters?" Review should be for research. Instead of simply listing and piling up the references, this review uses these references to comment on the theme of this paper.

Due to Google's withdrawal from China, I have encountered great difficulties in the process of searching literature on the internet. However, I have been still very tenacious from multiple sources to obtain research material I need: libraries, journals, books, and even friends abroad.

The key words I searched were as follows: water ice, hydroxyl radicals, methanol, hydroxyl groups, $\mathrm{CH}_{3} \mathrm{OH} / \mathrm{H}_{2} \mathrm{O}$ ratio, absorption, catalysts and Dr. Colaprete, etc.

\section{Literature Review}

\subsection{Is There Water Ice on Our Moon?}

Since 1961, some scientists have believed that water ice exists on the Moon (Watson et al. 1961). So, how do we evaluate the evidences provided by the scientists?

\subsubsection{Analysis of radar image data of the Antarctic region of the Moon}

According to the analysis of radar image data of the Antarctic region of the Moon in 1994, many researchers believed that there must be water in many permanent dark craters within the lunar Antarctic Aitken basin. The Moon Explorer has reinforced their view, suggesting more water ice also in the Moon's Arctic (Nozette et al. 1996).

However, some researchers believed that the existence of water ice can not be confirmed. The incidence angle of polar observation on Clementine is $82^{\circ} \sim 90^{\circ}$. Data will be abnormal caused by large incidence angle, due to shielding, diffraction and multiple scattering (Tsang., Kong., \& Shin 1985). Therefore, it is not certain that the increase in the polarizability of orbit is due to the Coherent Backscatter Opposition Effect (CBOE) or other scattering effects.

Although water ice can cause $\mathrm{CBOE}$, other scattering mechanisms (such as surface roughness, secondary reflection) can also explain the increase of Right Circular Polarization(RCP) in the lunar polar region. Therefore, the increase in the polarizability of Clementine's bi-static radar is not widely considered to be due to the presence of water ice in the lunar polar region(Stacy., Campbell., \& Ford 1997).

\subsubsection{Neutron spectral analysis of the Lunar Prospector}

Neutrons from the solar wind are constantly bombarding the surface of the Moon. But once they collide with hydrogen atoms, they lose their speed and become so-called thermal neutrons. The neutron spectrometer can be used to determine the hydrogen content on the Moon's surface.

The neutron spectrum data of Lunar Explorer show that the curves of the north and south poles of the Moon have an obvious turning point, which is exactly what those who expect to find water ice (actually hydrogen) on the Moon expect to see (Figure 1)
(Arnold 1979).

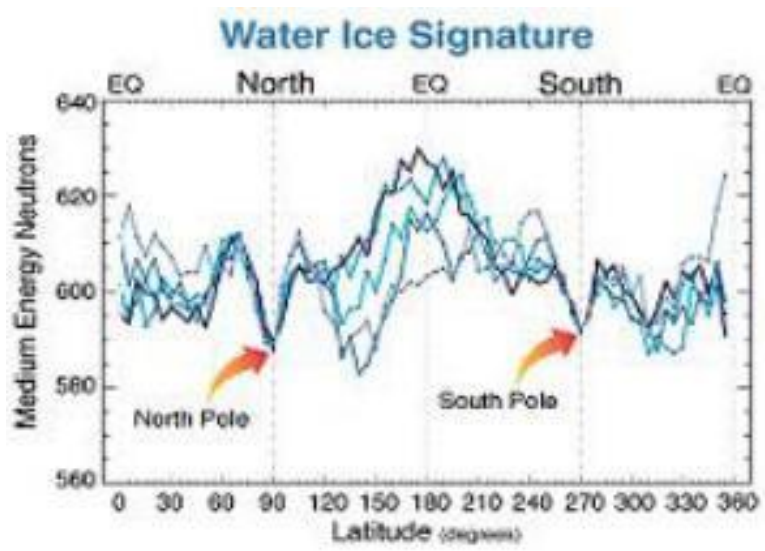

Figure 1. The Water Ice signal from the North and South Pole measured by the detector Neutron Spectrometer

However, some scholars think that the water ice loss rate caused by the impact vaporization of meteorites, the splashing of particles and the photoionization of interstellar hydrogen may exceed the amount of water ice transported to the Moon by comets (Cohen., Swindle., \& Kring 2000; Nimmo \& Korycansky 2012).

\subsubsection{Reflectivity anomaly}

The Clementine's bi-static radar measured radar echo energy and polarization patterns in the lunar polar regions, to look for the echo features of particular significance of frozen volatiles.

The frozen volatiles (including water ice) have the property of total internal reflection, which keeps the electromagnetic wave in the scattering signal in the original polarization mode. Freezing volatiles have lower transmission loss and higher average emissivity of corresponding electromagnetic waves than silicate rocks, which leads to higher echo energy, that is, can reflect more S-band electromagnetic waves than Moon's surface rocks (Ostro \& Shoemaker 1990).

American scientists believed that if the reflected energy curve peaks near $\beta=0$, there may be water ice there.

Figure 2 shows radar echo energy in four different orbits by the Clementine spacecraft. To some scientists, for the first time, there seems to be direct evidence of the presence of water ice at the Moon's poles (please note Orbit 234) (Nozette et al. 1996).

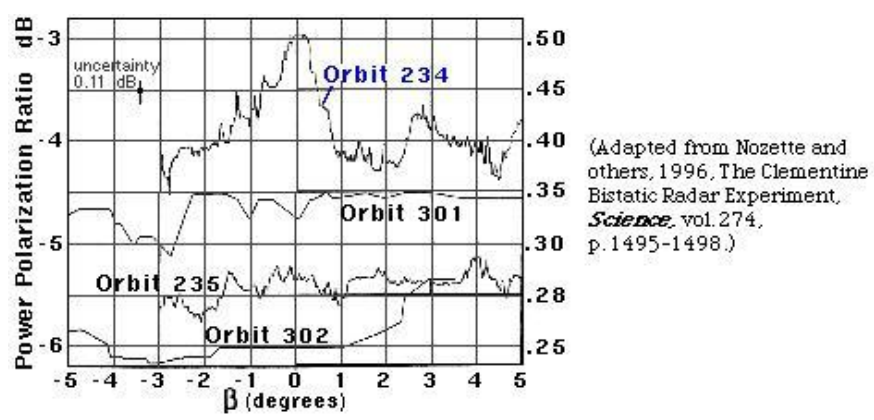

Figure 2. Radar wave reflection energy 
However, these scientists are not sure at heart. Otherwise, how could there be a great feat of hitting the lunar crater with the US Lunar Surveyor in order to finally determine whether there is really water ice?!

Yet the question remains whether the detected hydrogen abundance is actually water ice or pure hydrogen or even something else; hence, it is necessary to verify it.

\subsection{Verification}

\subsubsection{US Lunar Prospector}

On July 31, 1999, the US Lunar Prospector crashed into a dark crater near the Moon's South Pole as scheduled.

Water vapor or its photodissociation product"OH" (hydroxyl radicals), if accurately captured by spectral detection, would be ironclad proof of the presence of water ice in the weathering layer of the Moon.

Scientists had predicted that if water ice did exist at the impact point, the high temperature of the impact would immediately vaporize free water in the lunar soil and rocks, releasing at least 18 $\mathrm{kg}$ of water vapor. As the debris of the Moon reaches midair, the vapor will form a thin cloud that temporarily exists (lasts for 13 hours).

Hundreds of amateur and professional astronomers watched the impact with breath-holding everything from homemade telescopes to the world's best professional observatories.

However, no one could see or take a clear picture of the water vapor cloud column; neither the Earth Observatory nor the six space telescopes detected any water vapor or any other sign of water! The Moon's south pole seemed to have happened nothing in the minutes after the impact.

By 13 October 1999, the conclusion had finally come out. NASA and the University of Texas both announced that"the rover's impact on the Moon did not release detectable water ice signals."

The Washington Post also quoted some American scientists as saying that"the Moon's search for water has failed"; "There is no water in the lunar crater; the hydrogen detected by the lunar probe may only be pure hydrogen!"; "Is the hydrogen that has measured to be the solar wind protons or other?" (Crider \& Vondark 2000).

\subsubsection{Leo Meteors}

In fact, besides this man-made impact, a group of Leo meteors also hit the Moon on November 18, 1999. The meteors hit the Moon's north pole at a gentle angle, much like the Lunar Prospector's impact trajectory on July 31, 1999. As the result, no water ice evidence was obtained (Cudnik 2009).

\subsubsection{ESA Smart-1}

SMART-1, the European Space Agency (ESA)'s probe, successfully hit the Moon on September 3, 2006. Approximate impact coordinates were 34.4 S, 46.2 W (Sun 2018; ESA 2006a).
On 20 October 2006, the European Space Agency announced that "Astronomers using ground radio-telescopes have recently reported they were not able to detect a distinctive signature of thick deposits of ice in the area." We still do not know if this hydrogen is due to enhanced trapping of solar wind, or to the water ice brought on the Moon by the bombardment of comets and asteroids." (ESA 2006b).

Dr. Raj Sivalingam, the director of UK Space Policy and European Relations, said to me in 2006 via an email (Sun 2018): "Regarding the Moon, the presence of water ice at the South Pole is indeed controversial, and the earlier attempt to find it with Lunar Prospector was not definitive."

\subsubsection{Chandrayaan-1}

The researchers came to a conclusion that some frozen water should be in the darkest and coldest regions of the Moon after analyzing data from the Moon Mineralogy Mapper $\left(\mathrm{M}^{3}\right)$ spectrometer on the Indian satellite Chandrayaan-1 during 2008 2009.

However, I doubt this conclusion because there's a lot of uncertainties in their researches (Li \& Milliken 2017):

- " but as a whole, the $\mathrm{M}^{3}$ data do not provide clear evidence for magmatic water in the anorthositic crust."

- "the presence and mobility of molecular $\mathrm{H}_{2} \mathrm{O}$ on the lunar surface remain unclear based on the existing NIR data".

- "the existing $\mathrm{M}^{3}$ data are best interpreted as representing the presence and distribution of $\mathrm{OH}$ and provide no unambiguous evidence for the presence of $\mathrm{H}_{2} \mathrm{O}$ ".

A very important question is whether this "OH" is hydroxyl radicals of lunar water ice or hydroxyl groups from Moon's methanol?!

\subsubsection{US LCROSS}

Oct. 9, 2009, US LCROSS impacted into a permanently shadowed region of Cabeus crater near the Moon's south pole (Figure 3, by NASA) (Colaprete et al. 2010), creating "a two-part plume of material" from the bottom of the crater.

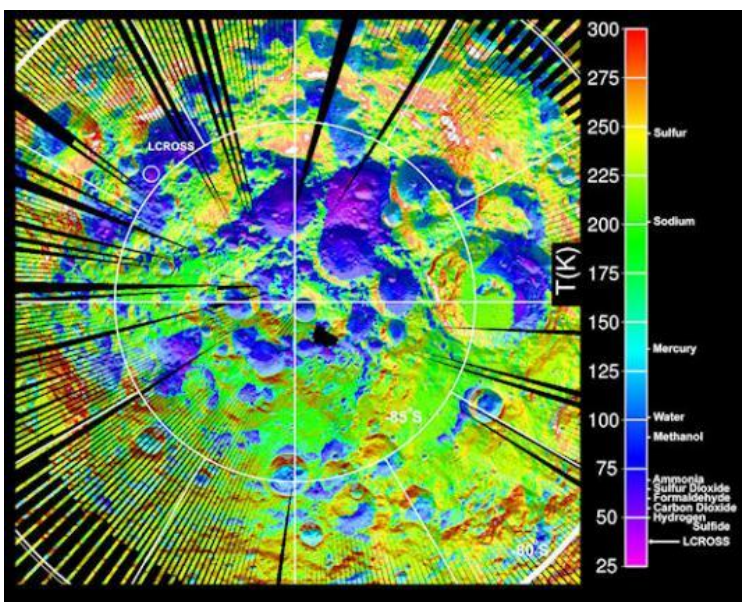

Figure 3 A surface temperature map of the lunar south pole 
It was said that "Near-infrared absorbance attributed to water vapor and ice and ultraviolet emissions attributable to hydroxyl radicals support the presence of water in the debris. The maximum total water vapor and water ice within the instrument field of view was $155 \pm 12$ kilograms." (Colaprete et al. 2010).

However, there were still some doubts due to the facts happened:

- "No ejecta plume was clearly detected at all", Anthony Colaprete himself had stressed at the time of the impact (NASA 2009).

- "We suspected there was water at the poles and learned for sure from LCROSS, but we now have evidence that there's water at mid latitudes." (Shekhtman 2019). LCROSS has unexpectedly been able to detect water at mid-latitudes up to 150 degrees Celsius, which eloquently proves that LCROSS detection is problematic, as ice is not stable on lunar surfaces that receive direct sunlight during part of the lunar diurnal cycle.

- The primary way that scientists find water is through remote sensing instruments that can identify what chemical elements things are made of based on the light they reflect or absorb. "But for that, you need a light source," Hurley said. "And by definition, these permanently shadowed regions don't have a strong one." (Shekhtman 2019)

. "But it wasn't clear from the LCROSS data where, exactly, that excavated ice originally lay - how much gray dirt once sat atop it. And, while several instruments have spotted tantalizing hints of exposed lunar ice over the years, these detections had remained unconfirmed until now. Previous observations indirectly found possible signs of surface ice at the lunar south pole, but these could have been explained by other phenomena, such as unusually reflective lunar soil," NASA officials wrote (Wall 2018).

- "If confirmed, this potential reservoir of frozen water on the Moon may be sufficiently massive to sustain long-term lunar exploration," said Noah Petro, Lunar Reconnaissance Orbiter Project Scientist at NASA's Goddard Space Flight Center in Greenbelt, Maryland (Steigerwald, B. \& Jones, N. 2019). Since it hasn't been confirmed, we can't take it lightly.

Small wonder some researchers says that the best way to find out for sure is to send spacecraft there to get some samples (Stacey 2019). "Until NASA astronauts get back to the Moon to dig up some soil, or the agency sends new instruments near the surface that can sniff out floating water molecules, the research team's theory about the influence of meteoroids on the environment inside shadowed craters could help chip away at some of the mysteries surrounding the Moon's water." (Shekhtman 2019)

\subsubsection{China "Chang'e-3"}

No water was found in the lunar soil chemical composition based on site exploration results of "Chang'e-3" (Zhang et al. 2015).

Moreover, "An unprecedented upper limit of the $\mathrm{OH}$ (Mgl) content in the lunar exosphere is obtained from the in-situ measurements carried out by LUT... The upper limit of $<10^{4} \mathrm{~cm}^{-3}$ derived for the $\mathrm{OH}$ radicals is lower than that derived from the HST low resolution spectroscopy by about two orders of magnitude, and is lower than that inferred from the mass spectra taken by the
Chandrayaan-1 mission... by about 6 orders of magnitude" (Wang et al. 2015). The lower the actual measurement of the $\mathrm{OH}(\mathrm{Mgl})$ content in the lunar exosphere, the less likely water is to exist on the surface of the Moon, of course.

The China State Administration of Science announced that there is no water on the Moon's Mare Imbrium (China State Administration of Science 2016) after two and a half years of field measurements since its successful landing on the Moon in December 2013.

\subsubsection{China's "Chang'e-4"}

On May 16, 2019, it was reported that the Yutu-2 deployed by China's Chang'e-4 lander revealed the presence of olivine and lowcalcium pyroxene on the floor of Von Kármán crater within the South Pole-Aitken Basin of Moon's far side (Choi 2019).

Moreover, on July 7, 2020, it was reported that Chinese scientists revealed analysis of weird substance found on the Moon's far side by Yutu 2 rover (Jones 2020).

However, in these reports, the water ice all did not be mentioned a word. If lunar water ice was found, how can they not announce it?

So it seems that what is it in the lunar polar craters remains unresolved.

\section{Body of the Text}

From the previous literature review, I found a problem that had not been paid attention to before: the widespread chemical role of lunar methanol.

The chemical mechanism of lunar methanol plays a key role in whether there is water ice on the Moon. So it seems that the special role of chemical sciences in the field of cosmic exploration should be highlighted.

Now, please note the temperature column on the right side of Figure 3. Blue mainly represents the"water ice and methanol". These blue areas are spread over the middle and high latitudes of the Moon. I think that one of the great achievements of US LCROSS is discovery of a large area of the methanol.

\subsection{Source of lunar methanol}

methanol $\left(\mathrm{CH}_{3} \mathrm{OH}\right)$ is an important interstellar molecule. $\mathrm{CH}_{3} \mathrm{OH}$ has been observed abundantly in both the gas phase and the solid state.

Solid methanol $\left(\mathrm{CH}_{3} \mathrm{OH}\right)$ is an important constituent of ices in the interstellar medium (ISM) (Dawes et al. 2016). $\mathrm{CH}_{3} \mathrm{OH}$ has been observed in comets (Wright et al. 2015) and on the surfaces of trans-Neptunian objects (Dalle et al. 2014). In dense molecular clouds, from which stars are formed, $\mathrm{CH}_{3} \mathrm{OH}$ is observed to be one of the most abundant constituents of ices after $\mathrm{H}_{2} \mathrm{O}$ and $\mathrm{CO}$ (Pontoppidan et al. 2004). 
It must be stressed that "spectra of Comet P/Swift-Tuttle indicate that the $\mathrm{v} 2$ and $\mathrm{v} 9$ bands of methanol comprise approximately $60 \%$ of the 'cometary organic feature'" (Hoban et al. 1993).

So, it seems that the widespread methanol on the Moon should mainly come from comets.

\subsection{Evidences of the presence of methanol on the Moon}

The evidences are as follows:

- "Carbon dioxide, methane, ethylene, and methanol were all found to be part of the LCROSS plume" (Colaprete et al. 2010; Farrell et al. 2017).

· "Other cometary volatiles are present (e.g., carbon dioxide, methane, sulfur dioxide, methanol, ethanol) in regolith of lunar Polar Regions" (Spudis 2013).

. "methanol $\left(\mathrm{CH}_{3} \mathrm{OH}\right)$ is one of the primordial volatiles contained within icy solids in the outer solar nebula...which suggests that $\mathrm{CH}_{3} \mathrm{OH}$ found in a persistently-shadowed lunar region probably formed through low-temperature surface chemistry on regolith" (Sekine et al. 2014).

It is necessary to point out that Qasim D. et al. studied formation of interstellar methanol ice prior to the heavy CO freeze-out stage, and got an important result: $\mathrm{CH}_{3} \mathrm{OH}$ formation is shown to be possible by the sequential surface reaction chain, $\mathrm{CH}_{4}+\mathrm{OH} \rightarrow \mathrm{CH}_{3}+$ $\mathrm{H}_{2} \mathrm{O}$ and $\mathrm{CH}_{3}+\mathrm{OH} \rightarrow \mathrm{CH}_{3} \mathrm{OH}$ at $10-20 \mathrm{~K}$ (Qasim et al. 2018), which much like the situation of the lunar polar craters ( $25 \mathrm{k}$ ).

That is to say, Chemical reactions still take place even in the ultralow temperatures such as in the lunar polar craters. Regardless of the ratio of methanol to water there, the end product of various chemical reactions is always methanol (see Figure 4).

The remaining water that might be present will be consumed in another reaction with methanol that produced continuously as shown in Figure 4 (Qasim D. et al. 2018), to produce vast quantities of molecular hydrogen, with a very high efficiency of lowtemperature hydrogen production using $\mathrm{Pt} / \mathrm{\alpha}-\mathrm{MoC}$ or $\mathrm{Pt} / \mathrm{C}$ catalysts (Lin et al. 2017), which see details in section 4.4.

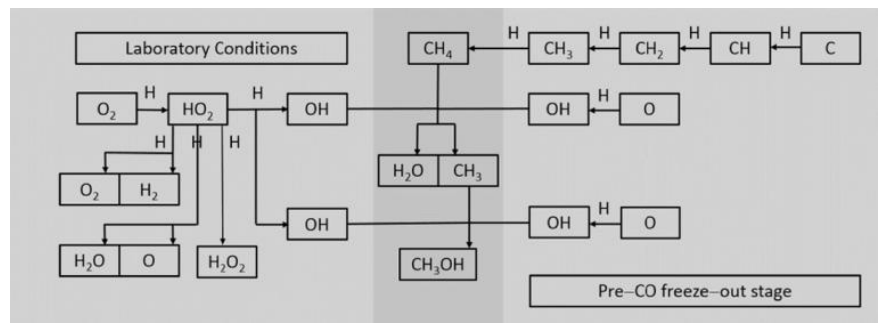

Figure 4 The formation of $\mathrm{CH}_{3} \mathrm{OH}$ in the pre-CO freeze-out stage (by Qasim D. et al. 2018)

\subsection{It is easy to confuse hydroxyl radicals and hydroxyl groups when interpreting $M^{3}$ data}

The "absorption strength" of hydroxyl radicals and hydroxyl groups are all $\sim 2.9 \mu \mathrm{m}$.
For example:

- The absorption strength of hydroxyl radicals is $\sim 2.9 \mu \mathrm{m}$ (Li \& Milliken 2017).

. "a broadband absorption at $2.9 \mu \mathrm{m}$ due to the presence of the hydroxyl groups in the calcium aluminate work" (Zhao et al. 2015).

. "The Moon Mineralogy Mapper $\left(\mathrm{M}^{3}\right)$ on Chandrayaan-1 has recently detected absorption features near 2.8 to 3.0 micrometers on the surface of the Moon. For silicate bodies, such features are typically attributed to hydroxyl- and/or water -bearing materials" (Pieters et al. 2009).

. "the overlapping of observed $\mathrm{CH}_{3} \mathrm{OH}$ vibrational absorption bands with $\mathrm{H}_{2} \mathrm{O}$ and silicate absorption features." (Dawes et al. 2016).

So it is easy to be confused hydroxyl radicals and hydroxyl groups when interpreting $\mathrm{M}^{3}$ data.

However, hydroxyl radicals and hydroxyl groups are two different concepts: hydroxyl radicals belong to the ion; whereas hydroxyl groups are the kind of functional groups in organic matter, at least exist in methanol (Gracia et al. 2008).

\subsection{A new theory}

The large amount of methanol exists on the Moon provides us with a new way of thinking:

hydroxyl radicals that LCROSS and Chandrayaan-1 claimed to have discovered on the Moon might be actually hydroxyl groups from Moon's methanol.

On our Earth, methanol can react with water to produce molecular hydrogen, with a very high efficiency of low-temperature hydrogen production using Pt/ $\alpha-M o C$ catalysts(Lin et al. 2017). The same catalysis in low-temperature applies to lunar polar craters that receive no sunlight.

Do these three chemical elements, Platinum(Pt), Carbon $(\mathrm{C})$ and Molybdenum(Mo), exist on the Moon? The answer is yes. The evidences are as follows:

Platinum(Pt) --- "Platinum is abundant on the Moon" (Shieber 2018).

Molybdenum(Mo) --- "The Russian Luna 24 mission discovered a single grain $(1 \times 0.6$ micrometer $)$ of pure molybdenum in a pyroxene fragment taken from Mare Crisium on the Moon" (New World Encyclopedia 2018).

Carbon(C) --- "Lava associated with lunar fire fountains contained significant amounts of carbon"(Saal et al. 2015). "There would appear to be $1.72 \%$ carbons from carbonaceous condrites" (Krahenbuhl et al. 1972). Moreover, Carbon may also be directly implanted in the lunar regolith from the solar wind (Bibring et al. 1974; Pillinger \& Eglinton 1977; Haskin \& Warren 1991).

In fact, besides Pt/ $\alpha-M o C$ catalysts, there is a better Pt/C catalyst. $\mathrm{Pt} / \mathrm{C}$ catalyst is the most active Hydrogen Evolution Reaction catalyst. Hydrogen Evolution Reaction can be realized at a voltage 
very close to the electromotive force of the thermodynamic reaction (Zhou et al. 2018). Since Pt and C are abundant on the Moon, this catalysis can be easily realized.

So the water brought by comets and asteroids and the one caused by solar wind has been exhausted by reacts with the widespread methanol on the Moon in the presence of Pt/ $\mathrm{a}-\mathrm{MoC}$ or $\mathrm{Pt} / \mathrm{C}$ catalysts. These reacts formed large amount of hydrogen, thus clarifying a question NASA raised that "Scientists have long speculated about the source of vast quantities of hydrogen that have been observed at the lunar poles" (NASA Content Administrator 2017).

So, what is the state of the vast quantities of hydrogen in the polar craters of the Moon now?

It found mid-winter, night-time surface temperatures inside the coldest craters - the south western edge of the floor of Hermite crater, the southern edges of the floors of Peary and Bosch craters in the northern polar region - can dip as low as minus $249^{\circ} \mathrm{C}(26 \mathrm{~K})$ (NASA Lunar Science Institute 2009), very close to the boiling point of hydrogen (minus $252.87^{\circ} \mathrm{C}$ ) and the melting point of hydrogen (minus $259.14^{\circ} \mathrm{C}$ ) on our Earth (Anon 2019a).

We can not exclude the presence of lower temperatures in the deeper locations of these lunar craters at North Pole, not to mention those at the bottom of lunar craters in the southern polar region where the sunlight never reached. If the temperature in those lunar polar craters above would be measured to further reduced by only $3.87^{\circ} \mathrm{C}$, it would have reached the boiling point of hydrogen (minus $252.87^{\circ} \mathrm{C}$ ) (if on the Earth), forming liquid hydrogen (Anon 2019a); and $10.14^{\circ} \mathrm{C}$ lower only, it would have reached the melting point of hydrogen (minus $259.14^{\circ} \mathrm{C}$ ) (if on the Earth), forming brown black solid molecular hydrogen appeared in snowflake patterns (Anon 2019b).

Given that the atmospheric pressure on the Moon surface is less than $1 / 10000$ of the one of the Earth surface, and its atmospheric density is 14 orders of magnitude smaller than that of the Earth (Ouyang 2005), so, what are the boiling point and the melting point of hydrogen on the surface of our Moon respectively? Is it necessary for the scientific community to conduct some relevant simulation experiments?

The existence of the solid molecular hydrogen or liquid molecular hydrogen in lunar polar craters can consistent with facts observed as follows:

1. Total internal reflections;

2. Increase in the same sense polarization;

3. Planar surface;

4. Maximum hydrogen abundance.

So, the solid molecular hydrogen or liquid molecular hydrogen in lunar polar craters is easy to confuse with water ice.

\section{Discussion}

I do not doubt the ability of LCROSS to detect $\mathrm{OH}$ from water, but only suspect that LCROSS is unable to distinguish between hydroxyl radicals from water ice and hydroxyl groups from Moon's methanol due to ignore their spectral identity. So, given the confusion caused by the spectral identity of lunar water ice with lunar methanol, some data showing that the water ice / methanol ratio in lunar polar craters is much greater than 1 should not be credible.

Due to Google's withdrawal from China, I have encountered great difficulties in the process of searching literature on the internet. Some evidences such as "to make a quantitative case for water exhaustion" can not be finished now. Fortunately, the purpose of my paper is only to raise a serious issue for discussion by the global scientific community. I am sure that the evidences will become more comprehensive as the discussion goes on.

Astronomy is the science of all mankind. Let's work together to find out whether there is water ice on our Moon.

\section{Conclusions}

Chemical reactions still take place even in the ultra-low temperatures such as in the lunar polar craters. Regardless of the ratio of methanol to water there, the end product of various chemical reactions is always methanol (see Figure 4). The remaining water that might be present will be consumed in another reaction with methanol that produced continuously as shown in Figure 4, to produce vast quantities of molecular hydrogen with a very high efficiency of low-temperature hydrogen production using Pt/a-MoC or Pt/C catalysts (Lin et al. 2017).

The absorption strength of hydroxyl radicals and hydroxyl groups are all $\sim 2.9 \mu \mathrm{m}$, so It is easy to confuse hydroxyl radicals and hydroxyl groups when interpreting $\mathrm{M}^{3}$ spectra data. However, hydroxyl radicals and hydroxyl groups are two different concepts: hydroxyl radicals belong to the ion; whereas hydroxyl groups are the kind of functional groups in organic matter, such as in methanol.

I put forward what scientists found in the lunar polar craters might be not hydroxyl radicals of water ice but only hydroxyl groups from lunar methanol and that all our previous misconceptions about water ice in the lunar polar craters might be due to the neglect of the widespread chemical role of lunar methanol. It is necessary to conduct in-depth research in this field in the future.

In addition, my paper also solves the following major problems:

- Where did the water to go, which brought by comets and asteroids to the Moon and the one caused by solar wind? $\rightarrow$ "the water has been exhausted by reacts with the widespread methanol on the Moon in the presence of Pt/ $\alpha-M o C$ or Pt/C catalysts";

- "lunar methanol can react with water brought by comets and asteroids to the Moon and the one caused by solar wind to produce large amount of molecular hydrogen, using $\mathrm{Pt} / \mathrm{\alpha}-\mathrm{MoC}$ or $\mathrm{Pt} / \mathrm{C}$ catalysts", thus clarifying a question NASA raised that "Scientists have long speculated about the source of vast quantities of hydrogen that have been observed at the lunar poles".

- The vast quantities of hydrogen found in lunar polar craters is most likely in the form of liquid or solid hydrogen. It's easy to confuse with water ice. 
In the field of whether there is water ice on the Moon, my paper explores almost all the major problems at the same time, thus constituting a system of its own.

\section{Conflicts of interest}

There are no conflicts to declare.

\section{Acknowledgements}

I thank Prof. Tim Horscroft for providing helpful comments.

\section{Notes and references}

1 Anon, 2019a. http://www.answers.com/Q/What is the melting point an $\mathrm{d}$ the boiling point of hydrogen in degrees Celsius

2 Anon, 2019b. https://baike.so.com/doc/30628093228446.html (in Chinese)

3 Arnold, J. R.,1979, Ice on the Moon. J. Geophys Res, 84, 5659-5668.

4 Barnes, J.J. Franchi, I.A. Anand, N. Tartèse, R. Starkey, N.A. Koike, N., et al., 2013, Accurate and precise measurements of the $\mathrm{D} / \mathrm{H}$ ratio and hydroxyl content in lunar apatites using NanoSIMS. Chemical Geology, 337, 48-55.

5 Barnes, J.J. Tartèse, R. Anand, N. McCubbin, F.M. Franchi, I.A Starkey, N.A., \& Russell, S.S., 2014, The origin of water in the primitive Moon as revealed by the lunar highlands samples. Earth and Planetary Science Letters, 390, 244-252.

6 Bibring J.P. et al. 1974. Simulation of lunar carbon chemistry: I. Solar wind contribution. In Proceedings of the Fifth Lunar Science Conference, Vol. 2, Lunar and Planetary Institute, Houston, pp 1747-1762

7 China State Administration of Science, 2016, Chang'e-3 announces latest scientific research results: Chang'e-3' Lunar Probe has confirmed that there is no water on the Moon. CCTV News, (in Chinese).

http://news.ifeng.com/a/20160731/49694003 0.shtml.

8 Choi, C.Q., 2019, What lies below the Moon's crust? China's Yutu-2 Rover may be the first to find out. Science \& Astronomy, https://www.space.com/moon-far-side-mantlechina-yutu-2-samples.html?utm source=sdcnewsletter\&utm medium=email\&utm campaign=20190516 $\underline{-s d c}$

9 Cohen, B. A. Swindle, T. D., \& Kring, D. A.,2000, Support for the lunar cataclysm hypothesis from lunar meteorite impact melt ages. Science, 290, 1754-1756.

10 Colaprete, A. Schultz, P. Heldmann, J. Wooden, D. Shirley, M. Ennico, K., et al. 2010, Detection of Water in the ICROSS Ejecta Plume. Science, 330, 463.

11 Crider, D. H., \& Vondark, R. R.,2000, The Solar Wind as a Possible Source of Lunar Polar Hydrogen Deposit. J Geophys Res, 105, 26733-26782.

12 Cudnik, B., Lunar Meteoroid Impacts and How to Observe Them, Springer-Verlag, New York, 2009. ISBN: 978-1-44190323-5 (Print) 978-1-4419-0324-2 (Online)

13 Dalle Ore C. et al., 2014. Asteroids, Comets, Meteors 2014. Proceedings of the conference held 30 June-4 July, 2014 in Helsinki, Finland, ed. K. Muinonen, et al., 2014, p. 398

14 Dawes A. et al., 2016. Using the C-O stretch to unravel the nature of hydrogen bonding in low- temperature solid methanol-water condensates. Phys. Chem. Chem. Phys., 18, 1245
15 ESA, 2006a, http://sci.esa.int/smart-1/39961-impactlanding-ends-smart-1-mission-to-the-moon/

16 ESA, 2006b, http://www.esa.int/Our Activities/Space Science/SMART1/Shackleton crater SMART-1 s search for light shadow and ice at lunar South Pole

17 ESA, 2015

http://www.esa.int/spaceinimages/Images/2015/11/Perspec tive view in Aurorae Chaos Ganges Chasma

18 ESA, 2017 http://www.esa.int/spaceinimages/Images/2017/07/Libya Montes topography

19 Farrell W. M. et al. 2017. The statistical mechanics of solar wind hydroxylation at the Moon, within lunar magnetic anomalies, and at Phobos. JGR Planets, 122(1), 269-289 https://doi.org/10.1002/2016JE005168

20 Gracia, L. Gonzalez-Navarrete P. Calatayud, M., \& Andres, J., 2008, A DFT study of methanol dissociation on isolated vanadate groups . Catalysis Today, 139, 214-220.

21 Haskin L. and Warren P. 1991. Lunar chemistry. In The Lunar Sourcebook: A User's Guide to the Moon, edited by Heiken G.H., Vaniman D., and French B.M., Cambridge University Press, Cambridge, UK, pp 357-474

22 Hoban S. et al. 1993. Infrared Observations of methanol in Comet P/Swift-Tuttle, Icarus, 105, 548-556

23 Jones A. 2020. Chinese scientists reveal analysis of weird substance found on the moon's far side by Yutu 2 rover, Space.com,

https://www.space.com/moon-far-side-yutu-2-rover-weirdsubstance-

analysis.html?utm source=Selligent\&utm medium =email\&u tm campaign=9155\&utm content $=S D C$ Newsletter + \&utm $t$ erm $=3586688 \& m$ i=7YU9ib7nCMNnNwGfNu Ezx\%2Be0xV\% 2BrKUjBpwX9D4yVzityJnrqD\%2B40Y7fnKqy9VAEQuCfWKp1r 7glwKoZjOylSmHzRcWmxapkxrRWPwU77F

24 Krahenbuhl, U. et al. 1972. in"The Apollo 15 Samples" (J. W. Chamberlain and C. Wathins, Eds.), p. 239. Lunar Science Institute, Houston.

$25 \mathrm{Li}, \mathrm{S}$, \& Milliken, R.E., 2017, Water on the surface of the Moon as seen by the Moon Mineralogy Mapper: Distribution, abundance, and origins. Science Advances, 3,9.

26 Lin, L.L, Zhou, W. Gao, R. Yao, S.Y. Zhang, X. Xu, W.Q., et al., 2017, Low-temperature hydrogen production from water and methanol using $\mathrm{Pt} / \alpha-\mathrm{MoC}$ catalysts. Nature, 544, 80-83.

27 Lucey P. et al. 2006. Rev. Mineral.Geochem. 60, 83. doi:10.2138/rmg.2006.60.2

28 Matthewman R. et al. 2015. The Moon as a Recorder of Organic Evolution in the Early Solar System: A Lunar Regolith Analog Study. Astrobiology, 15, 154-168. https://doi.org/10.1089/ast.2014.1217

29 NASA, 2009, LCROSS Impact: It's a Hit - But Was Anything Seen? Forum (in Chinese / English). http://bbs.astron.ac.cn/thread-63040-1-161.html

30 NASA Content Administrator, 2017. LCROSS Impact Data Indicate Water on Moon.

https://www.nasa.gov/mission pages/LCROSS/main/prelim water results.html

31 NASA Lunar Science Institute. 2009. LRO finds coldest place on the Moon, SSERVI. https://sservi.nasa.gov/articles/Irofinds-coldest-place-on-the-moon/

32 New World Encyclopedia, 2018, Molybdenum http://www.newworldencyclopedia.org/entry/Molybdenum

33 Nimmo, F., \& Korycansky, D.G., 2012, Impact-driven ice loss in outer Solar System satellites: Consequences for the Late Heavy Bombardment. Icarus, 219, 508 510.

34 Nozette, S. Lichtenberg, C.L. Spudis. P. Bonner, R. Ort, W. Malaret, E., \& Robinson, M., 1996, The Clementine bi-static Radar Experiment. Science, 274, 1495-1498. 
35 Ostro, S.J., \& Shoemaker, E.M. 1990, The extraordinary radar echoes from Europe, Ganymede, and Callisto: A Geological Perspective. Icarus, 85, 335-345.

36 Ouyang, Z.Y. (Chief editor), 2005, Introduction to lunar science: Beijing: China Astronavigation Press, (in Chinese).

37 Pieters C.M. et al. 2009. Character and Spatial Distribution of $\mathrm{OH} / \mathrm{H} 2 \mathrm{O}$ on the Surface of the Moon Seen by M3 on Chandrayaan-1. Science, 326(5952), 568-572. DOI: 10.1126/science. 1178658

38 Pillinger C. T. and Eglinton G. 1977. The chemistry of carbon in the lunar regolith. Philos Trans $R$ Soc Lond A 285, 369-377

39 Pontoppidan K, et al, 2004. Astron. Astrophys. 426, 925-940

40 Qasim D. et al., 2018. Formation of interstellar methanol ice prior to the heavy CO freeze-out stage. $A \& A, 612$, A83. https://doi.org/10.1051/0004-6361/201732355

41 Saal, A.,et al., 2015, Here's why fire fountains erupted on moon's surface. Nature Geoscience, Aug 25.

https://www.tribuneindia.com/news/sciencetechnology/story/124278.html

42 Sekine Y. et al. 2014. Impact chemistry of methanol: Implications for volatile evolution on icy satellites and dwarf planets, and cometary delivery to the Moon./carus, 243, 3947

https://www.sciencedirect.com/science/article/abs/pii/s00 19103514004503\#!

43 Shekhtman L., ( NASA's Goddard Space Flight Center, Greenbelt), 2019, Inside Dark, Polar Moon Craters, Water Not as Invincible as Expected, Scientists Argue, https://www.nasa.gov/feature/goddard/2019/inside-darkpolar-moon-craters-water-not-as-invincible-as-expectedscientists-argue

44 Shieber, J.,2018, With moon mining, space tourism and colonization on the horizon, Star Trek is only years away. Disrupt SF, https://techcrunch.com/2018/09/05/with-moonmining-space-tourism-and-colonization-on-the-horizon-startrek-is-only-years-away/?yptr=yahoo

45 Spudis P. D. 2013. Lunar Volatiles: The Next Steps in Their Characterization and Use. http://www.spudislunarresources.com/Papers/Spudis NLSI July 2013.pdf

46 Stacey K. 2019. New Research Sheds Light on the Ages of Lunar Ice Deposits. NASA: Earth's Moon https://www.nasa.gov/feature/goddard/2019/newresearch-sheds-light-on-the-ages-of-lunar-ice-deposits

47 Stacy, N.J.S. Campbell, D.B., \& Ford, P.G., 1997, Arecibo Radar Mapping of the Lunar Poles: A Search for Ice Deposits. Science, 276, 1527-1530.

48 Steigerwald, B. \& Jones, N., (NASA's Goddard Space Flight Center, Greenbelt, Md.) Aug. 3, 2019, The Moon and Mercury May Have Thick Ice Deposits, https://www.nasa.gov/feature/goddard/2019/moonmercury -ice

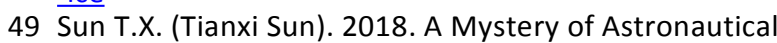
History: Additional Collision with Moon by SMART-1. American Journal of Geophysics, Geochemistry and Geosystems, 4 (3), 29-37 http://www.aiscience.org/iournal/paperlnfo/aj3g?paperld=4 211

50 Tartèse, R. Anand, M. McCubbin, F.M. Elardo, S.M. Shearer, C.K., \& Franchi, I.A., 2014, Apatites in lunar KREEP basalts: The missing link to understanding the $\mathrm{H}$ isotope systematics of the Moon. Geology, 42 (4), 363-366.

51 Tsang, L. Kong, J.A., \& Shin, R.T., 1985, Theory of Microwave Remote Sensing: In J A Kong(Editor): Wiley Series In Remote Sensing. Wiley -Interscience, New York.

52 Wall M., 2018, Water ice confirmed on the surface of the Moon for the $1^{\text {st }}$ time, SPACE, August 21, 2018 https://www.space.com/41554-water-ice-moonsurface-confirmed.html

53 Wang J. et al. 2015. An Unprecedented Constraint on water Content in the Sunlit Lunar ExospereSeen by Lunar-Based Ultraviolet Telescope of Chang'e-3 Mission. Planetary and Space Science, 109-110, 123-128

54 Watson, H. Murray, B.C., \& Brown, H., 1961, The behavior of volatiles on the lunar surface. J Geophys Res, 66, 3033-3045.

55 Wright I. et al, 2015. Science, 349, aab0673

56 Zhang, J.H. Yang, W. He, S. Lin, Y.T. Fang, G.Y. Li, C.L. et al. 2015, Volcanic history of the Imbrium basin: A close-up view from the lunar rover Yutu. PNAS, 112, 17.

57 Zhao H. F. et al. 2015. Effect of hydroxyl groups on infared transmittance of calcium aluminate glasses. Journal of the Chinese Ceramic Society. 43(2), 201-204

58 Zhou Y. Q. et al. 2018. Preparation of Pt/MoC and Its Performance in Electrolysis Hydrogen Evolution. Fine Chemicals (Online) Dol: 10.13550/j.jxhg.20180103. https://www.doc88.com/p-7975097401206.html 